\title{
Next-generation imaging in localized high-risk prostate cancer
}

\author{
Devin N. Patel $\mathbb{D}^{1} \cdot$ Lawrence I. Karsh ${ }^{2} \cdot$ Timothy J. Daskivich $\mathbb{D}^{3}$
}

Received: 22 February 2021 / Revised: 7 March 2021 / Accepted: 11 March 2021 / Published online: 31 March 2021

This is a U.S. government work and not under copyright protection in the U.S.; foreign copyright protection may apply 2021

Over the past decade, there has been an increased emphasis on avoiding overdetection and overtreatment of men with prostate cancer [1]. This has been achieved through improvements in risk stratification to better personalize decision-making. At the same time, and in part due to reduced overall prostate cancer screening rates, a greater proportion of men present with advanced disease at the time of diagnosis [2]. As such, there is a large unmet need to identify the subset of men with biologically aggressive disease who would best benefit from upfront local therapy. Toward this end, this month's issue of Prostate Cancer and Prostatic Disease highlights the work of Dekalo et al. in evaluating the use of next-generation imaging (NGI) with Ga-PSMA PET/CT to better risk stratify men with high-risk prostate undergoing radical prostatectomy.

Compared to 18-fluorodeoxyglucose, which accumulates in the bladder and obscures local recurrence, prostate cancerspecific tracers used for positron emission tomography (PET) may be better for prostate cancer. Three such tracers have emerged. These include F-18 fluciclovine (Axumin), which is an amino acid taken up in increased volume by cancer cells, F-18/C-11 choline, which targets cell membrane lipid biosynthesis that is increased in cancer cells, and prostate-specific membrane antigen (PSMA)-based tracers, which are comprised of a PSMA-targeting agent (PMSA11, DCGPyL) combined with a positron-emitting isotope (Gallium-68, fluorine F-18) [3].

PSMA-targeted tracers have emerged as the most sensitive and specific for detecting both locoregional and distant metastatic disease [4]. PSMA-based imaging has been studied

Devin N. Patel

d4patel@ucsd.edu

1 Department of Urology, The University of California, San Diego, CA, USA

2 The Urology Center of Colorado, Denver, CO, USA

3 Division of Urology, Cedars Sinai Medical Center, Los Angeles, CA, USA for both secondary staging in recurrent disease and primary staging for high-risk disease. For secondary staging, previous studies have indicated a sensitivity of $45 \%$ for PSMA-targeted radiotracers in men with PSA as low as $<0.5 \mathrm{ng} / \mathrm{mL}, 61 \%$ for those with PSAs $0.5-0.9 \mathrm{ng} / \mathrm{mL}$, and $78-95 \%$ for those with PSAs $>1.0 \mathrm{ng} / \mathrm{mL}$. Their use in this setting is endorsed by guidelines in men who may be candidates for salvage local or regional therapy [5]. Data for its use in primary staging is more limited. For this indication, most guidelines currently endorse use NGI to help clarify equivocal bone scan results. However, 2020 ASCO guidelines have broadened these indications to include men with negative conventional imaging [6]. In December 2020, the FDA approved Ga-68 PSMA-11 for PSMA-targeted PET imaging in men with prostate cancer, including men with high-risk disease who are candidates for local treatment, though the FDA approval was limited to two sites in California.

In this month's issue, Dekalo et al. examined rates of PSA persistence and recurrence in 149 men with National Comprehensive Cancer Network (NCCN)-defined high-risk prostate cancer who underwent Ga-68 PSMA-11 PET prior to robotic radical prostatectomy and extended bilateral lymph node dissection [7]. The authors found 13\% of men had $\geq 1$ preoperative positive lymph node on PSMA imaging. The sensitivity and specificity of PSMA imaging for detecting lymph node involvement were $68 \%$ and $95 \%$, respectively. Interestingly, all men with a preoperative lymph node $>1 \mathrm{~cm}$ on imaging had pathological lymph node involvement and PSA persistence following surgery. Following surgery, 84\% of men with PSMA-positive lymph nodes had PSA persistence compared to only $15 \%$ of men with negative preoperative PET. On multivariable analysis, including preoperative measures of PSA, biopsy grade group, and clinical stage, the presence of positive nodes on PSMA imaging had a remarkably strong association (odds ratio: 41.03 , 95\% CI: $10.43-230.08$ ) with postoperative PSA persistence.

In addition to results on men with PSMA-positive nodes, this paper also provides important information on men with NCCN high-risk disease and PSMA-negative nodes. 
Table 1 Sensitivity and specificity of PSMA-based imaging in primary staging.

\begin{tabular}{lllll}
\hline Author & Year & $N$ & Sensitivity & Specificity \\
\hline Maurer & 2015 & 130 & $66 \%$ & $99 \%$ \\
Budaeus & 2016 & 30 & $33 \%$ & $100 \%$ \\
Gupta & 2017 & 12 & $100 \%$ & $80 \%$ \\
Zhang & 2017 & 42 & $93 \%$ & $96 \%$ \\
van Leeuwen & 2017 & 30 & $64 \%$ & $95 \%$ \\
\hline
\end{tabular}

Among 111 men who were PET-node negative and with undetectable PSA following surgery, estimated 1- and 2year biochemical free recurrence survival rates were $87 \%$ (95\% CI: 80-94\%) and 76\% (95\% CI: 66-88\%). Despite a limited sample size, these numbers suggest a trend toward higher biochemical recurrence free survival rates than seen in all surgically treated men with high-risk disease, where 1and 2-year rates have been previously reported as low as $62 \%$ and $67 \%$, respectively [8]. Combined, these results indicate that a positive preoperative PSMA scan may identify subgroups of men at high risk of PSA persistence (who may benefit from clinical trials or multimodal approaches) and those with lower risk of PSA recurrence (who may benefit from standard radical prostatectomy). Of course, these results are observational in nature, and prospective study would be required for definitive conclusions about the optimal use of PSMA PET for triage of care.

Although the authors show that PSMA-based imaging for men with high-risk prostate cancer adds to traditional risk stratification parameters including PSA, stage, and grade, it remains unknown how NGI compares to conventional imaging in the setting of primary staging. Multiple studies have shown that PSMA PET/CT has a moderate sensitivity but very high specificity for dete-ction of nodal metastasis in intermediate-to-high-risk prostate cancer. Pooled analysis of five studies has shown a sensitivity and specificity of $77 \%$ and $97 \%$, respectively (Table 1). PSMA imaging has a high negative predictive value for enlarged but nonmetastatic lymph nodes. It also has the potential to detect small volume nodal disease. A retrospective study of 130 men with intermediate-to-high-risk disease showed that all missed lymph nodes occurred in patients with either metastasis from a PSMA-negative primary or a single micrometastatic node. A small prospective study of 30 patients was able to show that PSMA PET was able to detect disease in lymph nodes as small as $3-10 \mathrm{~mm}$, which falls below the size criteria for lymph node detection on conventional CT imaging and MRI [9].

We applaud the authors' efforts to improve the risk stratification of high-risk men with prostate cancer prior to surgery.
However, longitudinal comparative studies with survival endpoints are needed to determine the true overall benefit versus a perceived benefit due to reclassification. PSMAbased imaging has several important limitations. Further standardization in reporting criteria is needed for future clinical trials. Access to these imaging modalities is highly limited; there are currently only two sites in the United States with Ga-68 PSMA-1. Insurance coverage for these tests remains highly variable. Until these barriers are overcome, the development of comparative data to create and implement both clinically and cost-effective utilization parameters will be challenging.

\section{Compliance with ethical standards}

Conflict of interest The authors declare no competing interests.

Publisher's note Springer Nature remains neutral with regard to jurisdictional claims in published maps and institutional affiliations.

\section{References}

1. Daskivich TJ, Chamie K, Kwan L, Labo J, Palvolgyi R, Dash A, et al. Overtreatment of men with low-risk prostate cancer and significant comorbidity. Cancer. 2011;117:2058-66.

2. Barry MJ, Nelson JB. Patients present with more advanced prostate cancer since the USPSTF screening recommendations. J Urol. 2015;194:1534-6.

3. Liu W, Zukotynski K, Emmett L, Chung HT, Chung P, Wolfson R, et al. A prospective study of 18F-DCFPyL PSMA PET/CT restaging in recurrent prostate cancer following primary external beam radiotherapy or brachytherapy. Int J Radiat Oncol Biol Phys. 2020; 106:546-55.

4. Perera M, Papa N, Roberts M, Williams M, Udovicich C, Vela I, et al. Gallium-68 prostate-specific membrane antigen positron emission tomography in advanced prostate cancer-updated diagnostic utility, sensitivity, specificity, and distribution of prostate-specific membrane antigen-avid lesions: a systematic review and meta-analysis. Eur Urol. 2020;77:403-17.

5. Schaeffer E, Srinivas S, Antonarakis ES, Armstrong AJ, Bekelman JE, Cheng $\mathrm{H}$, et al. NCCN guidelines insights: prostate cancer, version 1.2021. J Natl Compr Canc Netw. 2021; 19:134-43.

6. Trabulsi EJ, Rumble RB, Jadvar H, Hope T, Pomper M, Turkbey $\mathrm{B}$, et al. Optimum imaging strategies for advanced prostate cancer: ASCO guideline. J Clin Oncol. 2020;38:1963-96.

7. Snir Dekalo JK, Mintz I, Fahoum I, Gitstein G, Keizman D. Preoperative 68Ga-PSMA PET/CT Defines a Subgroup of High-Risk Prostate Cancer Patients with Favorable Outcomes after Radical Prostatectomy and Lymph Node Dissection. Prostate Cancer Prostatic Dis. 2021 (in press).

8. Briganti A, Karnes RJ, Gandaglia G, Spahn M, Gontero P, Tosco $\mathrm{L}$, et al. Natural history of surgically treated high-risk prostate cancer. Urol Oncol. 2015;33:163.e7-13.

9. Patel DN, Nguyen C, Sirohi D, Falahatian V, Saouaf R, Luthringer $\mathrm{D}$, et al. Use of cylindrical coordinates to localize prostate cancers on MRI and prostatectomy pathology. Urol Oncol. 2017;35:673. e15-e20. 